\title{
Diagnostic Laparoscopy in Primary and Secondary Infertility
}

\author{
YEDIDYA HOVAV, ${ }^{1.2}$ ELIEZER HORNSTEIN,' MIRIAM ALMAGOR,' and CHAIM YAFFE ${ }^{1}$
}

Submitted: November 19, 1997

Accepted: May 7, 1998

Purpose: Our purpose was to compare the diagnostic efficacy of laparoscopy between primary and secondary infertility and to define a subgroup in which the positive finding.s are low.

Methods: A retrospective review of the cases of 206 infertile women who underwent laparoscopy was conducted.

Results: Eighty-two (39.8\%) patients were found to have evidence of pelvic disease-20 (22.2\%) with primary infertility and 62 (54.3\%) with secondary infertility. The ratio of positive findings in secondary infertility was significant in comparison with the positive findings in primary infertility. In only 3 of the 20 patients with primary infertility was there no history of an abdominal operation, pelvic inflammatory disease, or an abnormal hysterosalphingography. Of the 62 women who suffered secondary infertility and had positive findings in laparoscopy, 15 had no history of suspect findings.

Conclusions: The diagnostic yield of laparoscopy for primary infertility in the absence of indications of mechanical factors is low. Therefore the need for diagnostic laparoscopy in these cases should be reconsidered.

KEY WORDS: laparoscopy; primary infertility; secondary infertility.

\section{INTRODUCTION}

Laparoscopy should be the final procedure of any infertility investigation (1). However, more recently, an increasing number of clinicians have been using laparoscopy with hysteroscopy in the early stages of assessment of infertility (2). Although a simple technique in skilled hands, laparoscopy is not without complications, such as infections, hematomas, and injury to the bowel and to great vessels (3). Several studies reported

\footnotetext{
${ }^{1}$ Department of Obstetrics \& Gynecology, Bikur Cholim Hospital, Jerusalem, Israel.

${ }^{2}$ To whom correspondence should be addressed at Bikur Cholim Hospital, Department of Obstetrics and Gynecology, 5 Strauss Street, P.O.B. 492, Jerusalem, Israel.
}

that the incidence of unsuspected pelvic pathology found at laparoscopy is approximately $50 \%(4-7)$. It is reasonable to assume that mechanical problems are more frequent in secondary infertility than in primary infertility in couples that have no ovulation and sperm defects. However, we failed to find reports that make a distinction in the frequency of positive pathology findings at laparoscopy between primary and secondary infertility.

The aim of this retrospective study was to evaluate the yield of laparoscopy in primary infertility vs. secondary infertility and to define a subgroup that has a low chance of having abnormal findings at laparoscopy.

\section{MATERIALS AND METHODS}

Retrospective review of our infertility records during 1994-1996 identified 206 patients who underwent laparoscopy for investigation of infertility. All women had normal hormonal profiles and normal ovulatory cycles. Semen analysis in all cases was normal according to the World Health Organization criteria.

Patients were divided into two groups: 92 women suffering from primary infertility and 114 women with secondary infertility. The average age of women with primary infertility was $28.59 \pm 5.4$ years. The average duration of infertility was $5.4 \pm 4.1$ years. The average age of women with secondary infertility was $33.53 \pm$ 5.1 years. The average duration of infertility was 4.2 \pm 3.5 years. Hysterosalpingography (HSG) was performed in 82 patients with primary infertility and in 96 patients with secondary infertility. All the women who did not undergo HSG had a history of pelvic inflammatory disease (PID) or pelvic operation. Laparoscopy was performed under general anesthesia with endotracheal intubation using the double-puncture technique to maximize the exposure. All laparoscopies were performed by senior physicians who diagnosed endometriosis on inspection. HSGs were evaluated by 
a senior radiologist and then by a senior gynecologist. Both HSG and laparoscopy were carried out without complications.

Statistical analysis was performed using the chisquare test.

\section{RESULTS}

Of the 206 patients who underwent laparoscopy, $124(60.2 \%)$ had normal pelvic organs, while 82 $(39.8 \%)$ had evidence of pelvic disease.

Ninety-two patients were investigated for primary infertility. Pathology was observed in 20 (21.7\%) patients, the majority of whom (17 patients) had adnexal adhesions. All adhesions mentioned might potentially cause infertility (Table I). In approximately $50 \%$ of the abnormal cases, unilateral or bilateral tubal occlusion was observed. Endometriosis was found in three patients. The average age of women with pathologic findings was $31.4 \pm 4.2$ years.

In 114 patients with secondary infertility, diagnostic laparoscopy revealed pathology in $62(54 \%)$ patients. Pelvic adhesions were found in approximately $30 \%$ of the cases (Table I). Bilateral or unilateral tubal occlusion was observed in about a third of abnormal cases, and endometriosis was found in nine patients. The average age of women with pathologic findings in this group was $30.5 \pm 3.4$ years.

In the primary infertility group, 39 women had risk factors that could lead to mechanical problems. And indeed, in 17 of these women there were abnormal findings in laparoscopy. In the other group of 53 women with no previous risk factor, only 3 showed pathologic findings following laparoscopy. The percentage of women with normal pelvic organs in the primary infertility group $(78 \%)$ was significantly higher than that in the secondary infertility group $(P$ $<0.001)$.

Table I. Laparoscopic Findings in Patients with Primary and Secondary Infertility

\begin{tabular}{lcc}
\hline & \multicolumn{2}{c}{ Infertility } \\
\cline { 2 - 3 } \multicolumn{1}{c}{ Laparoscopy findings } & Primary (\%) & Secondary (\%) \\
\hline Pelvic adhesions & $17(18)$ & $35(30)$ \\
Bilateral tubal occlusion & $3(3)$ & $10(9)$ \\
Unilateral tubal occlusion & $6(6.5)$ & $9(10)$ \\
Endometriosis & $3(3)$ & $9(8)$ \\
Normal & $72(78)$ & $52(45)$ \\
Total & 92 & 114 \\
\hline
\end{tabular}

Table II. Distribution of Primary and Secondary Infertility

\begin{tabular}{lcc}
\hline \multicolumn{1}{c}{ Potential risk factor } & Primary & Secondary \\
\hline Abnormal HSG & 20 & 32 \\
PID & 2 & 11 \\
Ovarian cystectomy & 3 & 3 \\
Enuncleation of myoma & 1 & 3 \\
Appendectomy & 13 & 8 \\
Cesarean section & 0 & 10 \\
Ectopic pregnancies & 0 & 11 \\
Total & 39 & 78 \\
\hline
\end{tabular}

We attempted to evaluate the benefits of laparoscopy, by analyzing the history and the HSG results of the women who underwent the procedure. Table II summarizes the number of women who had PID, previous abdominal, gynecological operations, and abnormal HSG.

In the secondary infertility group, the presence or absence of a risk factor did not correlate with the findings in laparoscopy (Table III).

\section{DISCUSSION}

Laparoscopy is a very common procedure in the investigation of infertility. Among the United States board certified reproductive endocrinologists, $89 \%$ routinely order diagnostic laparoscopy for couples presenting with a primary complaint of infertility (2). In Canada in 1995, 63\% of infertile couples underwent diagnostic laparoscopy (8).

The purpose of this study was to evaluate the rate of abnormal laparoscopy in cases of previously unnoted risk factor to avoid having this procedure done routinely for all women.

The most common causes of pelvic pathology are PID and adhesions resulting from surgery. It is reasonable to expect that when such factors do not exist, the

Table III. Positive Findings in Laparoscopy According to Their Gynecological History and HSG Results

\begin{tabular}{lcc}
\hline \multirow{2}{c}{ Group } & \multicolumn{2}{c}{ Infertility } \\
\cline { 2 - 3 } & Primary (\%) & Secondary (\%) \\
\hline Risk factor present & $17 / 39(43)^{a}$ & $47 / 78(60)$ \\
No risk factor & $3 / 53(6)$ & $15 / 36(41)^{b}$ \\
\hline${ }^{a} P<0.001$ compared with the no-risk-factor group in primary \\
infertility. \\
${ }^{h} P<0.001$ compared with the no-risk-factor group in primary \\
infertility.
\end{tabular}

Journal of Assisted Reproduction and Genetics, Vol. 15, No. 9, 1998 
pathologic incidence would be much lower. Likewise, when the HSG is normal, the incidence of pathologic findings is relatively low.

We found that the incidence of normal laparoscopy in primary infertility is significantly higher than in secondary infertility. However, this difference is expressed only in women evaluated as having no risk factor. In cases with previous risk factors, there is no significant difference between primary and secondary infertility.

It is noteworthy that in secondary infertility with no apparent risk factors, the rate of abnormal laparoscopy was relatively high $(41 \%)$. These results may indicate that pregnancy, abortion, delivery, etc., also play a significant role in asymptomatic PID and ultimately in mechanical problems.

One might argue that the higher incidence of pelvic abnormalities in the group of patients with secondary infertility could be related to the patients' ages. In our study, however, although as a whole, the secondary infertility group was slightly older (33, compared to 28 ), those within the group who showed abnormal findings were actually younger than those with similar pathology in the primary infertility group.

In view of these results, we suggest that in the evaluation of primary infertility, diagnostic laparoscopy can be delayed when no risk factors exist.
The diagnostic yield of laparoscopy for secondary infertility in the absence of indications of mechanical factors is substantial (54\%). The need for diagnostic laparoscopy in secondary infertility is therefore well founded.

\section{REFERENCES}

1. Speroff L. Glass RH. Kase NG: Investigation of the infertile couple. In Clinical Gynecologic Endocrinology and Infertility. Baltimore. Williams \& Wilkins, 1989, pp 513-546

2. Glatstein IZ. Harlow BL. Hornstein MD: Practice patterns among reproductive endocrinologists: the infertility evaluation. Fertil Steril. 1997: 67:443-45!

3. Brosens IA, Gordon AG: Tubal Infertility. London-New York, Gower Medical, 1989

4. Abdulrahman WE: Laparoscopic evaluation of apparently normal infertile women. Aust NZ Obstet Gynaecol 1994:34:440-442

5. Adelusi B, Al-Nuaim L: Accuracy of hysterosalpingography and laparoscopic hydrotubation in diagnosis of tubal patency. Fertil Steril 1995;6.3: 1016-1020

6. Goldenherg RL, Magendantz: Laparoscopy and the infertility evaluation. Obstet Gynecol 1976;47:410-4/4

7. Wood GP: Laparoscopic examination of the normal infertile woman. Obstet Gynecol 1983;62:642-643

8. Collins JA, Feeny D, Gunby J: The cost of infertility diagnosis and treatment in Canada in 1995. Hum Reprod 1997;12:951-958 